\title{
SEGMENTED COMPRESSIVE SENSING
}

\author{
Vahid Abolghasemi, Saeid Sanei, Saideh Ferdowsi, Foad Ghaderi and Allan Belcher
}

\author{
abolghasemiv@cf.ac.uk
}

\begin{abstract}
This paper presents an alternative way of random sampling of signals/images in the framework of compressed sensing. In spite of usual random samplers which take $p$ measurements from the input signal, the proposed method uses $M$ different samplers each taking $p_{i}{ }^{\prime}(i=1,2,3 \ldots M)$ samples. Therefore, the overall number of samples will be $q=M \bar{p}^{\prime}$. Using this method a variable sampling criterion based on the content of the segments is achievable. Following this idea, the calculated measurement (or sensing) matrix is also more incoherent in columns comparing to other conventional methods which is a desired feature. Our experiments show that the reconstructed signal using this method has a better SNR and is more robust compared to the systems using one sampler.
\end{abstract}

Index Terms - compressed sensing, incoherency, $\ell_{1}$-norm, random sampling, sparsity, thresholding

\section{INTRODUCTION}

In recent years many researchers have been working on a new concept in the area of signal processing and information theory called Compressed Sensing (CS) [1]. It states that under some conditions we can violate Nyquist rate rule when sampling signals and take fewer measurements and still preserve most information in the signal.

The most important condition for the input signal in this framework is sparsity. The sparsity requires a significant number of signal samples to be zero. This condition can exist in any known basis (e.g. Wavelet, Fourier, etc.). There have been many researches on finding the best sparse representation of a signal [2] for years which can be employed in CS applications. Assuming the signal can be transformed to a sparse domain, a linear combination of this sparse signal is computed as measurements. This can be easily done by multiplying a $p \times n$ matrix by the sparse version of input signal ( $p$ is the number of measurements and $n$ is the total number of input samples). The advantage is that $p<<n$ and hence the number of coefficients to be saved or sent is very small.

There are some important issues here that should be considered wisely; what type of matrix (dictionary) can be used for taking measurements? How can we reconstruct the original signal from these measurements? The majority of works in recent years have been devoted to answer these two im- portant questions. Different structures for the measurement matrix have been proposed in the literature. These include random Gaussian, Bernoulli ( \pm 1 entries), partial Fourier basis and wavelet [3].

The reconstruction algorithms can be generally categorized into greedy algorithms [4] [5] and relaxation optimization methods [2]. Having least number of measurements with least reconstruction error is always desired. Obviously, sparsity of the input signal plays an important role in defining the allowed number of measurements.

In this paper a segment-based technique for designing a measurement matrix with higher incoherency in columns is proposed. The idea is very simple and efficient. We take $q$ measurements which output from $M$ different random samplers with different rates. The total measurements taken are then combined together and make the measurement matrix. The aim is to reach to a measurement matrix with most incoherency. Comparing the present work with other existing algorithms showed outperforming of the proposed method.

The rest of the paper is as follows. In section 2 an overview of CS theory is presented. Section 3 describes the proposed method in designing the measurement matrix. Simulation results are presented in section 4 . Finally the paper is concluded in section 5 .

\section{COMPRESSED SENSING THEORY}

To mathematically express the compressed sensing idea, suppose the input signal $\vec{x}$ is $k$-sparse over a dictionary (e.g. time, pixel, Fourier, Wavelet). It means that there are only $k<<n$ non-zero samples in that sparse signal. $\vec{x}$ can be represented in the sparse domain using the simple linear matrix multiplication:

$$
\vec{x}=\Psi \vec{\alpha}
$$

If $\vec{x}$ is assumed to be an $n \times 1$ vector, then the dimensions of $\Psi$ and $\vec{\alpha}$ are $n \times n$ and $n \times 1$, respectively. The measurements or observations are obtained by multiplying $\Phi$ by equation (1). Here, the number of rows in $\Phi$ which is in fact the number of measurements, called $p$, is preferred to be as small as possible:

$$
\vec{y}=\Phi \Psi \vec{\alpha}
$$


If for example we assume that $\vec{x}$ is sparse in Fourier domain, then $\Psi$ is, Fourier basis and $\vec{\alpha}$ is the $k$-sparse coefficient vector. There are some points that should be noted here. First, the selected number of measurements is highly related to the level of sparsity; more sparsity allows us to take smaller $p$. It has been shown that $p_{\text {min }}=C \log (n / k)$ [1] where $C$ is a constant. Second, in order to have a successful CS system, $\Phi$ and $\Psi$ must be as incoherent as possible in their columns. This refers to the theory of Uniform Uncertainty Principle (UUP) [3]. The connection between this theory and compressive sampling is that the sparsifying matrix should be as much incoherent as possible with the sensing matrix. The coherency measure between the sensing basis $\Phi$ and the presenting basis which is sometimes called "mutual coherency" is [3]:

$$
\begin{gathered}
\mu(\Phi, \Psi)=\sqrt{n} \max _{1 \leq k, j \leq n}\left|\left\langle\phi_{k}, \psi_{j}\right\rangle\right| \\
1 \leq \mu \leq \sqrt{n}
\end{gathered}
$$

In fact, mutual coherency is the largest correlation between any two columns of $\Phi$ and $\Psi$. From the compressibility point of view, we always seek for $\Phi$ and $\Psi$ pairs which are as less coherent as possible. There are many pairs with good incoherency in literature [3]. However, it is proven that random matrices are largely incoherent with any fixed bases. This is a very important property that allows us to choose measurement matrix non-adaptively [3]. One idea is to take random measurements from the input signal with a rate lower than Nyquist rate. This should be done in the domain in which the signal is not sparse. Another significant property in compressive sampling signals is theory of Restricted Isometry Property (RIP) that should be considered as an effective factor in robustness of CS [3]. For each integer $k=1,2, \ldots$ the isometry constant $\sigma_{k}<<1$ of a matrix $\Phi$ is defined as the smallest number such that:

$$
\left(1-\sigma_{k}\right)\|\vec{x}\|^{2} \leq\|\Phi \vec{x}\|^{2} \leq\left(1+\sigma_{k}\right)\|\vec{x}\|^{2}
$$

Where $\|$.$\| refers to \ell_{2}$-norm.

This equation holds for all $k$-sparse vectors [3]. Suppose now we only have the measurements $\vec{y}$ and the measurement matrix $\Phi$ in hand; we want to recover signal $\vec{x}$ from $\vec{y}$. Obviously, we are dealing with an underdetermined system with more unknowns than knowns $(n>p)$. In order to solve such a system appropriately, we have to add sparsity constraint to the system. In other words, we are always seeking for the sparsest solution either in sample domain $(\vec{x})$ or in sparse transform domain $(\vec{\alpha})$ which is proven to be unique [1]. The most straightforward solution is $\ell_{0}$-norm which calculates the number of non-zero elements in the sparse signal expressed by:

$$
\hat{\vec{\alpha}}=\arg \min \|\vec{\alpha}\|_{0} \text { s.t. } \quad \vec{y}=\Phi \Psi \vec{\alpha}=\mathbf{D} \vec{\alpha}
$$

Although solving equation (5) is an intractable problem in general, some greedy techniques such as MP (Matching
Pursuit) and OMP (Orthogonal MP) [5] have been reported which use an iterative soft thresholding (shrinkage) technique to find the sparsest vector. This is achieved either using $\ell_{0}$ or $\ell_{1}$-norm which is defined by replacing $\|\cdot\|_{0}$ with $\|\cdot\|_{1}$ in equation (5). It has been shown that we can have the sparsest unique solution by applying $\ell_{1}$-norm optimization as well. It leads to a convex optimization problem which is solved by linear programming, mainly [2]. BP (Basis Pursuit) is one of these methods.

In general, greedy methods are fast but not very accurate. In contrast, the relaxation optimization techniques are more accurate but require very high computation time. Generally, decreasing the computational complexity as well as increasing the accuracy, especially in the case of noisy data, is still an open issue.

\section{MEASUREMENT MATRIX CALCULATION}

One of the most important parts in a CS system that has to be well designed is the measurement matrix. Although random sampling allows us to easily take a small number of samples $p$ as the measurements, it is not so simple in practice to find the best measurement matrix $\Phi$ with smallest $p$ leading to an accurate reconstruction. However, to guarantee the accurate reconstruction for a variety of input signals we must increase $p$ which is not desired. There have been few researches in designing an optimum measurement matrix in literature but they are not all efficient [6] [7]. Most of them suffer from high computational complexity and are application dependant. In [6] Elad uses an iterative thresholding method to reduce the incoherency $\mu$. It attempts to decrease off-diagonal elements of Gram matrix which is $\mathbf{G}=(\Phi \Psi)^{T}(\Phi \Psi)$. The results are encouraging but the complexity is high and the method can not be applied to large scale problems (e.g. images).

In this paper a new and simple technique to design the measurement matrix is proposed. It is not claimed that the proposed method is efficient in general, but it shows good empirical results for different real and synthetic signals. The proposed method has connection with the theory of distributed CS [8] but it also has some differences.

It is known that designing $\Phi$ (or potentially D) in euqation (5) with maximum independency in its columns increases the performance of reconstruction procedure. The reason is that in this case we can reconstruct the original signal with least possible atoms leading to a sparse solution. On the other hand, it is shown that we are allowed to draw this dictionary in different ways; Random Gaussian, Bernoulli ( \pm 1 entries), partial Fourier or Wavelet basis [3]. The criterion to decide about the efficiency or inefficiency of matrix $\mathbf{D}$ is "mutual coherency" which has been discussed earlier in section 2. Apart from the mentioned methods in defining sensing matrix, there have been recently a few works on finding the sensing matrix, not necessarily randomly or partially from a known basis [6][7]. They try to optimize an initially selected matrix in 
order to increase incoherency in columns. The proposed algorithm also aims at finding a $\mathbf{D}$ with smallest $\mu$.

The idea in present work is that, instead of calculating a measurement matrix for the entire input at once, we divide the input signal into $M$ segments and then take $p_{i}^{\prime}(i=$ $1,2, \ldots M)$ samples variably from each segment. These measurements are then placed together and constitute $\Phi$. If we define the average number of measurements per segment as $\bar{p}^{\prime}$, the total number of measurements we take will be $q=M \bar{p}^{\prime}$, which can be less than $p$ in general. Algorithm 1 shows the pseudocode for the proposed method. Literally speaking, the objective is to satisfy $q \leq p$ while the quality of the results is superior. It should be noted that although we see a better performance using the proposed method, $q$ can not be less than the minimum value defined in the literature which is on the order of $C \log (n / k)$. The advantage here is that $\mathbf{D}$ has a smaller $\mu$ than those reported in the literature. Moreover, variability in taking measurements can lead to a wised sampling, and still having non-coherent samples.

\section{Algorithm1}

\section{- Initialization}

$-\vec{x}_{n \times 1}$ : Input signal in non-sparse domain

$-p_{i}{ }^{\prime}$ : Number of measurements for each signal segment $(i=$ $1,2, \ldots M)$

$-M$ : Number of segments

\section{- Sampling}

- Divide the input signal into $M$ segments, and take $p_{i}{ }^{\prime}$ random samples of each (overall samples are $q=M \bar{p}^{\prime}$ )

- Concatenate $M$ samplers appropriately and call it $\Phi$

- Compute $\vec{y}_{q \times 1}=\Phi_{q \times n} \vec{x}_{n \times 1}$

\section{- Reconstruction}

- Reconstruct $\vec{\alpha}$ using one of the recovery techniques.

\section{EXPERIMENTAL RESULTS}

The first experiment has been carried out by making a synthetic sparse signal in frequency domain, converting it to time (non-sparse) domain and then randomly sample it based on different $M$ and $p^{\prime}$. We then reconstruct this signal by solving $\ell_{1}$ optimization problem using BP (Basis Pursuit) method. The codes have been used from $\ell_{1}$ - magic toolbox [9] which is a well designed and simple MATLAB toolbox available online for solving the convex optimization problems, mainly based on standard interior-point methods. It has been adapted to be applicable for CS problems. Fig. 1 displays the results for a signal of length $n=120$ and only 5 non-zero samples which we call it 5 -sparse. In this experiment $\bar{p}^{\prime}=4$ and $M=5$. Hence, the total number of measurements $q$ is $5 \times 4=20$. Fig. 1(a) and (b) are the input signal representation in non-sparse (time) and sparse (frequency) domains, respectively. Fig. 1(c) is the reconstructed signal by solving $\ell_{1}$ optimization using $\mathrm{PB}$ and after sensing by the proposed method. Fig. 1(d), (e) and (f) are the reconstructed signals using BP [9], Matching Pursuit [5] and $s \ell_{0}$ (smoothed- $\ell_{0}$ ) [10], respectively and with traditional sensing method (random sampling). $s \ell_{0}$ is a fast optimization method which approximates the $\ell_{0}$-norm of a vector by a smooth function $F_{\sigma}($.$) , where \sigma$ determines the quality of approximation [10]. As it is seen from Fig. 1, the proposed system accurately reconstructs the input signal while others do not.

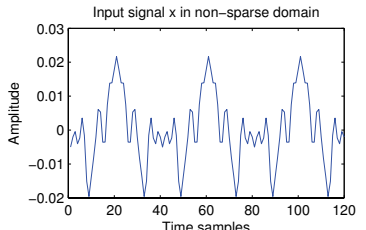

(a)

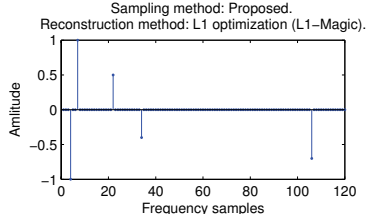

(c)

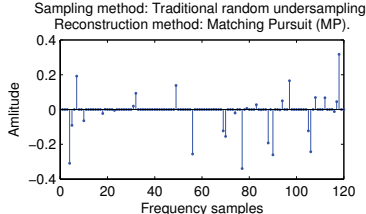

(e)

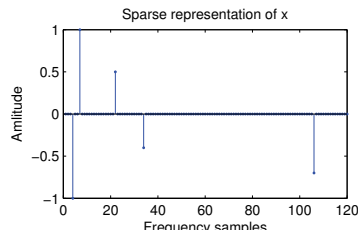

(b)

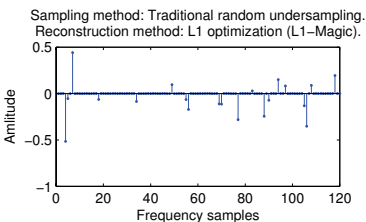

(d)

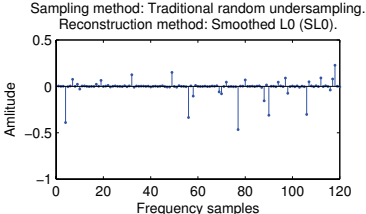

(f)
Fig. 1. Results of different reconstruction techniques by applying proposed and traditional measurement matrices.

The reason of obtaining such a good reconstructed signal is that the proposed method causes the measurement matrix to be very incoherent in columns. In this experiment the value $\mu$ obtained using the proposed method is 1.1468 while the value obtained using conventional methods is 1.4952 . The computed SNR parameter is also depicted in Table 1. It is seen that the proposed method has significantly a better performance.

Table 1. SNR value for different experiments

\begin{tabular}{|c|c|c|c|c|}
\hline Sensing technique & Proposed & \multicolumn{3}{|c|}{ Random sampling } \\
\hline Recovery algorithm & BP & BP & MP & $s \ell_{0}$ \\
\hline SNR(dB) & 97.35 & 39.82 & 38.69 & 37.61 \\
\hline$\mu$ & 1.1468 & 1.4952 & 1.4952 & 1.4952 \\
\hline
\end{tabular}

In the second experiment, a fixed number of measurements $q=25$ is selected for a signal with the length of $n=120$. Then the number of non-zeroes in the sparse signal is changed from 1 to 8 . The reconstruction error for different sensing matrices $\Phi$ (proposed, traditional random matrix, $s \ell_{0}$ 


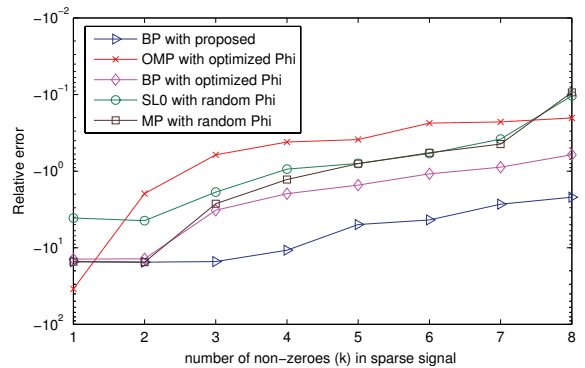

Fig. 2. CS reconstruction error for different methods as a function of number of non-zero samples (k).

[10], optimized $\Phi[6])$ and different reconstruction methods are computed. The results have been shown in Fig. 2. As expected, with increasing $k$, the error deteriorates. On the other hand, the robustness of the proposed method is clearly seen. In average, the error in the proposed method is considerably less than the error caused by other methods.

In the third experiment a grayscale image is used as an input (Fig. 3(a)). The reconstruction results for different values of $M$ and $\bar{p}_{i}{ }^{\prime}$ have been depicted in Fig. 3(b) and (c). It is seen that the proposed sensing matrix outperforms other methods.

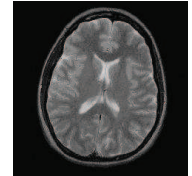

(a)

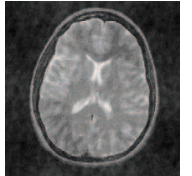

(b)

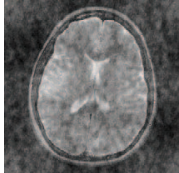

(c)
Fig. 3. Results for a $500 \times 500=250000$ pixel image, using Fourier domain as a sparse domain, reconstruction method: BP (a) Input image. Reconstructed image for (b) $M=100, \bar{p}^{\prime}=1500$ (c) $M=1$ and $\bar{p}^{\prime}=150000$

We also evaluated the performance of the proposed algorithm against different values for $M$ and $\bar{p}^{\prime}$. In this experiment a 10-sparse signal is selected as the input. Then it is fed to the system for 3 different values of $M$. The total number of measurements $q$ in all three experiments is increased by a step of 5 and the SNR value is recorded. Fig. 4 displays a graph related to this experiment. It is clear that with increase in $M$, the performance is improved. However, choosing a very large $M$ which leads to selection of a very small $p^{\prime}$ causes the reconstruction methods to fail. Hence, choosing a reasonable combination of number of segments and measurements is always required. This can be done based on the prior knowledge about the nature of input signal.

\section{CONCLUSION}

An improvement to designing the measurement matrix in compressive sampling of signals has been presented. The proposed method first divides the input signal into $M$ segments.

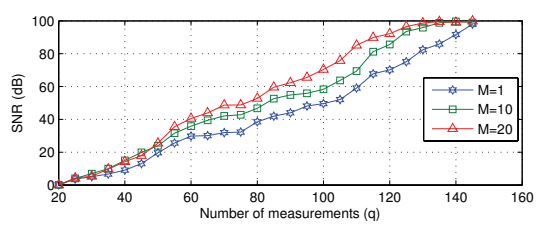

Fig. 4. SNR in $\mathrm{dB}$ for different $M$ for a 10-sparse signal with total number of samples $n=150$

Then it takes $p^{\prime}$ samples from each segment. In this way we have $\bar{p}^{\prime}$ average number of samples per segment. The advantage is that the resulting measurement matrix has a lower coherency in its columns comparing to the existing ones. This leads to a better reconstruction result and allows us to take fewer measurements for sending or processing the data. The experiments confirmed the ability of the proposed method to improve the performance.

\section{REFERENCES}

[1] D. Donoho, “Compressed sensing," IEEE Trans. on Information Theory, vol. 52, pp. 1289-1306, 2006.

[2] D. Donoho, "For most large underdetermined systems of linear equations the minimal $\ell_{1}$-norm solution is also the sparsest solution," Communications on pure and applied mathematics, vol. 59, no. 6, pp. 797-829, 2006.

[3] E. J. Cands and M. B. Wakin, "An introduction to compressive sampling," IEEE Signal Processing Magazine, vol. 25, no. 2, pp. 21-30, March 2008.

[4] S. F. Cotter and B.D. Rao, "Sparse channel estimation via matching pursuit with application to equalization," IEEE Trans. on Communications, vol. 50, no. 3, pp. 374-377, March 2002.

[5] S. Mallat and Z. Zhang, "Matching pursuits with timefrequency dictionaries," EEE Trans. on Signal Processing, vol. 41, no. 12, pp. 3397-3415, December 1993.

[6] M. Elad, "Optimized projections for compressed sensing," IEEE Trans. on Signal Processing, vol. 55, no. 12, pp. 1537 - 1541, December 2007.

[7] V. D. M. Nhat, S. Challa, DucVo, and S. Y. Lee, "Efficient projection for compressed sensing," Seventh IEEE/ACIS International Conference on Computer and Information Science (ICIS), pp. 322-327, May 2008.

[8] M. F. Duarte, S. Sarvotham, D. Baron, M.B. Wakin, and R.G. Baraniuk, "Distributed compressed sensing of jointly sparse signals," Conference Record of the Thirty-Ninth Asilomar Conference on Signals, Systems and Computers, pp. 1537 - 1541, October 2005.

[9] E. Candes and J. Romberg, " $\ell_{1}$-magic: Recovery of sparse signals via convex programming," [Online]. Available: http://www.acm.caltech.edu/l1magic/.

[10] H. Mohimani, M. Babaie-Zadeh, and C. Jutten, "A fast approach for overcomplete sparse decomposition based on smoothed $\ell_{0}$ norm," IEEE Trans. on Signal Processing, vol. 57, no. 1, pp. 289-301, January 2009. 